Results Linear blood flow velocity (LBFV) in the vein of Galen was assessed intraoperatively in all patients. The operative treatment stage was completed when the LBFV in the vein of Galen decreased to $25-50 \%$ of the initial value. Echo imaging showed that pulmonary hypertension was significantly reduced in 10 patients during the postoperative period, except in a patient with a choroidal subtype of vein of Galen malformation. Favorable outcomes were observed for eight $(72.7 \%)$ patients. Two $(18.8 \%)$ patients developed complications, both being caused by intracranial hemorrhage. Two patients who had been operated on died (16.6\%).

Conclusions Endovascular treatment of vein of Galen aneurysmal malformations in neonates with severe pulmonary hypertension is feasible. This technique reduces the severity of pulmonary hypertension, thus lowering the risk for developing critical heart failure or multiple organ failure, and allows patients to cross the critical line on their way to survival. To be treated using endovascular surgery, patients need to undergo meticulous selection, and surgical treatment should be used only if they have the mural subtype of vein of Galen malformations. The initially poor patient's condition (the Bicêtre score $<8$ ), the choroidal subtype of malformation, and concurrent cardiac malformations are the factors preventing the favorable outcome of endovascular treatment of vein of Galen malformations in neonates.

Disclosures P. Seleznev: None. K. Orlov: None. V. Berestov: None. A. Gorbatykh: None. D. Kislitsyn: None. T. Shayakhmetov: None. N. Strelnikov: None.

\section{0-030 LONG-TERM CLINICAL AND RADIOGRAPHIC FOLLOW-UP OF PURE ARTERIAL MALFORMATIONS}

${ }^{1} S$ Oushy ${ }^{*},{ }^{2} \mathrm{~W}$ Brinjikji, ${ }^{2} \mathrm{H}$ Cloft, ${ }^{1} \mathrm{R}$ Vine, ${ }^{1} \mathrm{G}$ Lanzino. ${ }^{1}$ Neurosurgery, Mayo Clinic, Rochester, MN; ${ }^{2}$ Radiology, Mayo Clinic, Rochester, MN

10.1136/neurintsurg-2020-SNIS.30

Introduction Pure arterial malformations (PAM) are rare vascular lesions characterized by dilated, tortuous arterial loops without venous shunting. The natural history of these lesions remains unclear. We report the long-term clinical and radiological outcomes in the largest series of patients with PAM.

Methods Retrospective review at a tertiary academic referral center for patients with a PAM. Follow-up clinical and radiological data were collected and analyzed for clinical symptoms and radiographic changes.

Results Twenty-five patients met the inclusion criteria. The mean age at presentation was $30.9 \pm 14$ years. Nineteen $(76 \%)$ patients were female and six $(23.1 \%)$ were male. Eleven (44\%) patients had $\geq 1$ symptom at presentation, of which, only $3(12 \%)$ could be linked to PAM. The most common symptom was headache $(n=8)$. PAMs involved a single vessel in $16(64 \%)$ cases and $\geq 2$ vessels in 9 cases (36\%). Fifteen $(60 \%)$ lesions were isolated to the anterior circulation compared to $6(24 \%)$ in the posterior circulation. The most frequently involved vessel was the supraclinoid internal cerebral artery (36\%). An associated saccular aneurysm was present in $32 \%$ of patients. Ten lesions were partially calcified. The mean radiographic and clinical follow-up was 21.4 \pm 26.6 months and $44.5 \pm 34.8$ months, respectively. None of the patients developed new symptoms related to their lesion or radiographic progression over the duration of follow-up.

Conclusion PAMs are rare incidental vascular lesions found most frequently in young, asymptomatic females. PAMs have a benign clinical and radiographic natural history and are best managed conservatively with serial imaging.

Disclosures S. Oushy: None. W. Brinjikji: None. H. Cloft: None. R. Vine: None. G. Lanzino: None.

\section{0-031 CEREBRAL ANEURYSM TREATMENT TRENDS IN NATIONAL INPATIENT SAMPLE 2007-2016: ENDOVASCULAR THERAPIES FAVORED OVER SURGERY}

${ }^{1} \mathrm{~A}$ Wang ${ }^{*},{ }^{2} \mathrm{~J}$ Campos, ${ }^{3} \mathrm{G}$ Colby, ${ }^{4} \mathrm{~A}$ Coon, ${ }^{2} \mathrm{~L}$ Lin. ${ }^{1}$ College of Osteopathic Medicine of the Pacific, Western University of Health Sciences, Pomona, CA; ${ }^{2}$ University of California Irvine Medical Center, Orange, CA; ${ }^{3}$ University of California Los Angeles, Los Angeles, CA; ${ }^{4}$ The Carondelet Neurological Institute, St. Joseph's Hospital, Tucson, AZ

\subsection{6/neurintsurg-2020-SNIS.31}

Introduction The neuroendovascular space continues to evolve with innovative devices. Flow diversion is the newest endovascular technique for cerebral aneurysm treatments since Food and Drug Administration (FDA) approval in 2011. We conducted a national database study to investigate any change in clinical practice patterns.

Methods United States National Inpatient Sample (NIS) databases from 2007 to 2016 were queried for hospital discharges with unruptured aneurysm (UA) and/or ruptured aneurysm (RA) patients receiving surgical clipping (SC) and/or endovascular treatments (EVT) using the International Classification of Diseases (ICD) Ninth Revision-Clinical Modification (CM), ICD Tenth Revision-CM, and ICD Tenth Revision-Procedure Coding System. Patient demographics, hospital characteristics and clinical outcomes were reviewed. Sub-group analysis was performed for treatment differences in 2007-2011 versus (vs.) 2012-2016 timeframes.

Results A total of 39,282 hospital discharges were identified with significant increase in EVT (UA: SC $\mathrm{n}=7,847$ vs. EVT $\mathrm{n}=12,797, \mathrm{p}<0.001 ;$ RA: SC $\mathrm{n}=8,108$ vs. EVT $\mathrm{n}=10,530$, $\mathrm{p}<0.001)$. Hospitals in the South demonstrated the most significant EVT use irregardless of aneurysm status (UA: SC $\mathrm{n}=258.5 \pm 53.6$ vs. EVT $\mathrm{n}=480.7 \pm 155.8, \mathrm{p}<0.001 ; \mathrm{RA}$ : SC $n=285.6 \pm 54.3$ vs. EVT $n=393.3 \pm 102.9, p=0.003)$ From 2007-2011, there was no significant difference in the mean number of cases for the treatment modalities (UA: SC $\mathrm{n}=847.4 \pm 107.7$ vs. EVT $\mathrm{n}=1,120.4 \pm 254.1, \mathrm{p}=0.21$; RA: SC $\mathrm{n}=949.4 \pm 52.8$ vs. EVT $\mathrm{n}=1,054.4 \pm 219.6, \mathrm{p}=0.85)$. The 2012-2016 period demonstrated significant increase in mean number of cases treated endovascularly for both UA and RA (UA: SC $n=722.0 \pm 43.4$ vs. EVT $n=1,439.0 \pm 419.2$, $\mathrm{p}<0.001 ;$ RA: SC $\mathrm{n}=672.2 \pm 61.4$ vs. EVT $\mathrm{n}=1,051.6 \pm$ 330.2, $\mathrm{p}=0.02$ ).

Conclusions As technological innovations continue to advance the neuroendovascular space, the standard of care for cerebral aneurysm treatments further shift towards endovascular therapies over open surgical approaches in the United States.

Disclosures A. Wang: 1; C; Western University Summer Research Fellowship Award. J. Campos: None. G. Colby: 2; C; Stryker Neurovascular, Medtronic. A. Coon: 2; C; Stryker Neurovascular, Medtronic, MicroVention. 4; C; InNeuroCo. L. Lin: 2; C; Stryker Neurovascular, MicroVention, Cerenovus. 